Revista Energia na Agricultura

ISSN 1808-8759

\title{
VARIAÇÃO DIMENSIONAL DAS TÁBUAS DE Eucalyptus grandis DURANTE ALGUNS NÍ- VEIS DE TEMPO E TEMPERATURA DE TERMORRETIFICAÇÃO ${ }^{1}$ FRED WILLIANS CALONEGO ${ }^{2} \&$ ELIAS TAYLOR DURGANTE SEVERO ${ }^{3}$
}

RESUMO: O presente estudo teve como objetivo avaliar os efeitos do tempo e da temperatura de termorretificação na variação em espessura das tábuas de Eucalyptus grandis. Para tanto, foram utilizadas tábuas provenientes de toras de Eucalyptus grandis, com cerca de 30 anos de idade, da Floresta Estadual de Santa Bárbara, pertencente ao Instituto Florestal do Estado de São Paulo. Cada tábua foi seccionada de modo a fornecer peças destinadas para a termorretificação, com temperaturas finais de $180^{\circ} \mathrm{C}$ e $200^{\circ} \mathrm{C}$ durante 2,5 , 3,0 e 4,0 horas. Os resultados mostraram que: (1) o material termorretificado com $200^{\circ} \mathrm{C}$ durante 3 a 4 horas apresentou aumento da espessura entre $0,698 \mathrm{~mm}$ e $0,874 \mathrm{~mm}$ devido ao surgimento de rachaduras internas; (2) o material termorretificado com $180^{\circ} \mathrm{C}$ durante 2,5 horas apresentou diminuição de $0,177 \mathrm{~mm}$ na sua espessura e não ocorreu presença de rachaduras internas.

Palavras-chave: Termorretificação da madeira, Rachaduras internas, Eucalyptus grandis.

\footnotetext{
${ }^{1}$ Trabalho extraído de tese defendida no Programa de Pós-graduação em Agronomia - Energia na Agricultura;

${ }^{2}$ Engenheiro Florestal Dr., Departamento de Recursos Naturais/Ciências Florestais, Universidade Estadual Paulista, Fazenda Experimental Lageado, s/n - Caixa Postal 237 - Botucatu, SP - CEP 18603-970, e-mail: fwcalonego@ig.com.br

${ }^{3}$ Professor Adjunto, Departamento de Recursos Naturais/Ciências Florestais, Universidade Estadual Paulista, Fazenda Experimental Lageado, s/n - Caixa Postal 237 - Botucatu, SP - CEP 18603-970, e-mail: severo@fca.unesp.br
} 


\section{DIMENSIONAL CHANGE OF EUCALYPTUS GRANDIS TIMBER DURING SOME LEVELS OF RETIFICATION TEMPERATURE AND TIME}

SUMMARY: The objective of this study was evaluate the effects of retification ${ }^{\circledR}$ temperatures and times on thickness variation from Eucalyptus grandis timber. Boards from logs of 30-year-old Eucalyptus grandis trees, from São Paulo Forest Institute in Santa Barbara, Brazil, were thermally modified at $180^{\circ} \mathrm{C}$ for 2.5 hours, at $200^{\circ} \mathrm{C}$ for 3.0 hours and $200^{\circ} \mathrm{C}$ for 4.0 hours. The results showed that: (1) the thermally modified wood at $200^{\circ} \mathrm{C}$ by 3.0 to 4.0 hours showed increased thickness between $0.698 \mathrm{~mm}$ and $0.874 \mathrm{~mm}$ due to the internal cracks, (2) the thermally modified wood at $180^{\circ} \mathrm{C}$ by 2.5 hours has a decreased from 0,177 $\mathrm{mm}$ in thickness and the absences of internal cracks.

Keywords: Retification®; Cracks; Eucalyptus grandis.

\section{INTRODUÇÃO}

Muitos processos de termorretificação da madeira são descritos na literatura. Em geral, eles apresentam em comum o fato da madeira ser exposta a temperaturas próximas à $200^{\circ} \mathrm{C}$ durante várias horas (WASKETT e SELMES, 2001; RAPP, 2001; ROUSSET et al., 2004; METSÄ-KORTELAINEN et al., 2005).

Em escala laboratorial, a termorretificação da madeira é realizada por alguns pesquisadores em estufa elétrica, na qual a madeira é submetida ao ar aquecido com temperaturas entre $100^{\circ} \mathrm{C}$ e $200^{\circ} \mathrm{C}$ durante um período que varia de 2 a 24 horas, para que ocorra a degradação das hemiceluloses e a condensação dos componentes em um polímero complexo e modificado, com o intuito de melhorar a sua durabilidade biológica e a sua estabilidade dimensional (BEKHTA e NIEMZ, 2003; JUODEIKIENĖ e MINELGA, 2003; POPPER et al., 2005; BRITO et al., 2006).

Rousset et al. (2004) verificaram que a termorretificação à $200^{\circ} \mathrm{C}$, durante 1 hora, diminuiu significativamente a higroscopicidade da madeira de Populus robusta entre $40 \%$ e $60 \%$. Contudo, o tratamento não alterou a permeabilidade da madeira, pois, não gerou rachaduras internas e não afetou a morfologia dos poros. 
Segundo Unsal e Ayrilmis (2005), após o aquecimento da madeira de Eucalyptus camaldulensis, entre $120^{\circ} \mathrm{C}$ e $180^{\circ} \mathrm{C}$, durante 2 a 10 horas, houve um decréscimo na sua massa específica a $12 \%$ de umidade com o aumento da temperatura e do tempo de exposição ao tratamento. A madeira tratada por 10 horas com $150^{\circ} \mathrm{C}$ e $180^{\circ} \mathrm{C}$ apresentou, respectivamente, reduções de $7,1 \%$ e $10,0 \%$ na propriedade física estudada.

Madeiras de Pinus sylvestris e de Picea abies submetidas à termorretificação com $170^{\circ} \mathrm{C}, 190^{\circ} \mathrm{C}$, $210^{\circ} \mathrm{C}$ e $230^{\circ} \mathrm{C}$, por 3 horas, apresentaram relações diretas entre a perda de massa e o aumento da temperatura. O alburno de Pinus quando submetido a tais temperaturas, apresentou, respectivamente, perda de massa na ordem de $1,8 \%, 4,1 \%, 6,7 \%$ e $11,8 \%$, e o cerne reduções equivalentes a $5,4 \%, 5,9 \%, 6,9 \%$ e 11,0\%. Para o alburno de Picea, as perdas de massa foram de 1,8\%, 3,3\%, 6,6\% e $10 \%$ quando submetidas a $170^{\circ} \mathrm{C}, 190^{\circ} \mathrm{C}, 210^{\circ} \mathrm{C}$ e $230^{\circ} \mathrm{C}$ e, no cerne, as respectivas reduções apresentaram-se em torno de 3,6\%, 5,3\%, 6,9\% e 11,4\% (METSÄ-KORTELAINEN et al., 2005).

Alén et al. (2002) e Sundqvist et al. (2006), em seus respectivos estudos, concluíram que a madeira de Picea abies e a de Betula pubescem termorretificadas com temperaturas entre $180^{\circ} \mathrm{C}$ e $225^{\circ} \mathrm{C}$, durante 2 a 8 horas, e entre $160^{\circ} \mathrm{C}$ e $200^{\circ} \mathrm{C}$, durante 1 a 4 horas, apresentaram maior perda de massa com o aumento do tempo de exposição e da temperatura do tratamento.

As madeiras de Picea abies termorretificadas por 4 horas, com temperaturas de $180^{\circ} \mathrm{C}$ e $225^{\circ} \mathrm{C}$, apresentaram perda de massa de $1,5 \%$ e $10,8 \%$; enquanto as tratadas com as mesmas temperaturas, durante 6 horas, apresentaram perda de massa de 1,7\% e 12,5\% (ALÉN et al., 2002). As madeiras de Betula pubescem tratadas por 2,5 horas, com temperaturas de $160^{\circ} \mathrm{C}$ e $200^{\circ} \mathrm{C}$, apresentaram perda de massa de $20 \%$ e $48 \%$ (SUNDQVIST et al., 2006).

Hakkou et al. (2005a) salientaram que a madeira de Fagus sylvatica termorretificada durante 8 horas, sob temperatura de $180^{\circ} \mathrm{C}$, apresentou perda de massa em torno de $7 \%$. Com o aumento da temperatura, houve um aumento progressivo da perda de massa até um valor máximo de $35 \%$ na madeira tratada à $260^{\circ} \mathrm{C}$.

Hakkou et al. (2005b) estudaram o efeito da temperatura, entre $40^{\circ} \mathrm{C}$ e $260^{\circ} \mathrm{C}$, durante 8 horas, na perda de massa das madeiras das folhosas Fagus sylvatica e Populus nigra e das coníferas Pinus sylvestris e Abies Alba. Os autores concluíram que a perda de massa tornou-se significativamente apreciável a partir dos $180-200^{\circ} \mathrm{C}$ para as folhosas e, já ocorreu, a partir de $50^{\circ} \mathrm{C}$ nas coníferas. No primeiro grupo de madeiras, a degradação das hemiceluloses é o principal responsável pela perda de massa, e, no segundo grupo, os extrativos voláteis também interferem neste fenômeno, pois, são degradados em baixas temperaturas.

Juodeikienè e Minelga (2003) estudando o efeito do aquecimento entre $60^{\circ} \mathrm{C}$ e $120^{\circ} \mathrm{C}$ durante 24 a 96 horas, concluíram que o tratamento com maiores tempo e temperatura proporcionou diminuição da higroscopicidade da madeira de Pinus sp. e um aumento na sua estabilidade dimensional. A madeira não 
tratada apresentou teores de umidade de equilíbrio higroscópico (TUE) de 8,44\% e 13,30\%, respectivamente, quando exposta em ambientes interno e externo. As tratadas à $60^{\circ} \mathrm{C}$ durante 24 horas apresentaram $7,78 \%$ e $12,36 \%$, e as tratadas à $120^{\circ} \mathrm{C}$ por 96 horas apresentaram 5,92\% e 10,86\% de TUE nos ambientes interno e externo. Os autores ainda verificaram que, a madeira não tratada apresentou variação nas dimensões radial e tangencial de $1,550 \mathrm{~mm}$ e $2,835 \mathrm{~mm}$, a tratada com $60^{\circ} \mathrm{C}$ durante 24 horas apresentou variações dimensionais de 1,490 e $2,800 \mathrm{~mm}$ e a tratada com $120^{\circ} \mathrm{C}$ por 96 horas apresentou variações em torno de 1,350 e $2,635 \mathrm{~mm}$.

Entretanto, Unsal e Ayrilmis (2005) afirmam que há pouca informação sobre o efeito dos tratamentos térmicos nas propriedades tecnológicas da madeira de Eucalyptus, principalmente sobre as propriedades físicas.

Assim, o objetivo do presente trabalho foi avaliar o efeito do tempo e da temperatura de termorretificação na variação em espessura das tábuas de Eucalyptus grandis.

\section{MATERIAL E MÉTODOS}

Para realização desse estudo foi utilizada a madeira proveniente de 12 árvores de Eucalyptus grandis, com 30 anos de idade. O material foi coletado na Floresta Estadual de Santa Bárbara, pertencente ao Instituto Florestal do Estado de São Paulo e localizado no município de Águas de Santa Bárbara - SP. Nesse estudo foi utilizado somente material proveniente de toras de diâmetro médio de 30 a $35 \mathrm{~cm}$ e que apresentava massa específica básica de $0,599 \pm 0,031 \mathrm{~g} / \mathrm{cm}^{3}$.

\subsection{Preparo do material}

De cada árvore foram retiradas toras, com cerca de 2,9 metros de comprimento, e estas foram desdobradas em tábuas com $28 \mathrm{~mm}$ de espessura através de cortes tangenciais na serraria do Horto Florestal de Mandurí, pertencente ao Instituto Florestal do Estado de São Paulo e localizado no município de Mandurí - SP.

Posteriormente, toda a madeira foi seca em uma estufa piloto de secagem convencional do Laboratório de Secagem e Preservação de Madeiras, pertencente à FCA-UNESP de Botucatu-SP, com capacidade para aproximadamente $2,5 \mathrm{~m}^{3}$ de madeira serrada. Somente as tábuas que continham a medula foram selecionadas para a realização desse estudo. 


\subsection{Termorretificação das tábuas}

As tábuas centrais (àquelas que continham a medula) foram cortadas em peças menores com 0,50 m de comprimento, sendo que foram descartadas as regiões com rachaduras e nós e o material contido até $30 \mathrm{~cm}$ das extremidades.

Cada peça, com teor de umidade de $10,0 \%$, teve os seus topos selados com borracha de silicone vermelho (resistente a temperaturas de até $316^{\circ} \mathrm{C}$ ) e foi empilhada de modo gradeado em uma estufa elétrica.

O material foi aquecido com uma temperatura inicial de $100^{\circ} \mathrm{C}$ com o intuito de diminuir o seu teor de umidade para cerca de 3\% e evitar possíveis problemas de expansão de vapor de água e rompimento das paredes celulares. Após esse período, as cargas de madeira foram submetidas a uma taxa de elevação de temperatura de $1,34^{\circ} \mathrm{C} / \mathrm{min}$, em função das recomendações de Rousset et al. (2004), Alén et al. (2002) e Sundquist et al. (2006), até um conjunto de tempos e temperaturas finais, conforme mostra a Tabela 1.

Após os tratamentos de termorretificações, a estufa foi desligada e as peças de madeira permaneceram no seu interior em resfriamento natural até atingirem a temperatura de $30^{\circ} \mathrm{C}$.

Tabela 1 - Temperaturas e tempos de termorretificação utilizados nas tábuas de Eucalyptus grandis.

\begin{tabular}{cccc}
\hline Temperatura de Termorretificação $\left({ }^{\mathbf{0}} \mathbf{C}\right)$ & \multicolumn{2}{c}{ Tempo de Termorretificação (horas) } \\
\hline Inicial & Máxima & $\begin{array}{c}\text { na Taxa de Elevação } \\
\left(\mathbf{1 , 3 4} \mathbf{C}^{\mathbf{0}} \mathbf{C} / \text { minuto }\right)\end{array}$ & na Temperatura Máxima \\
\hline 100 & 200 & 1,24 & 4,0 \\
\hline 100 & 200 & 1,24 & 3,0 \\
\hline 100 & 180 & 1,00 & 2,5 \\
\hline
\end{tabular}

\subsection{Avaliação da qualidade das tábuas termorretificadas}

A avaliação das tábuas após os tratamentos de termorretificação indicou que não houve nenhum tipo de empenamento e/ou de rachaduras superficiais ou de topo. Assim, ao término dos tratamentos, a variação em espessura das tábuas de Eucalyptus grandis foi determinada.

Como mostra a Figura 1, todas as tábuas tiveram as suas espessuras mensuradas em pontos prédeterminados da região periférica do fuste com o auxílio de um micrômetro com precisão de $0,001 \mathrm{~mm}$. 
Essas medições foram realizadas antes e após as termorretificações com $200^{\circ} \mathrm{C}$ de temperatura durante 4 horas, com $200^{\circ} \mathrm{C}$ de temperatura durante 3 horas e/ou com $180^{\circ} \mathrm{C}$ de temperatura por um período de 2,5 horas.

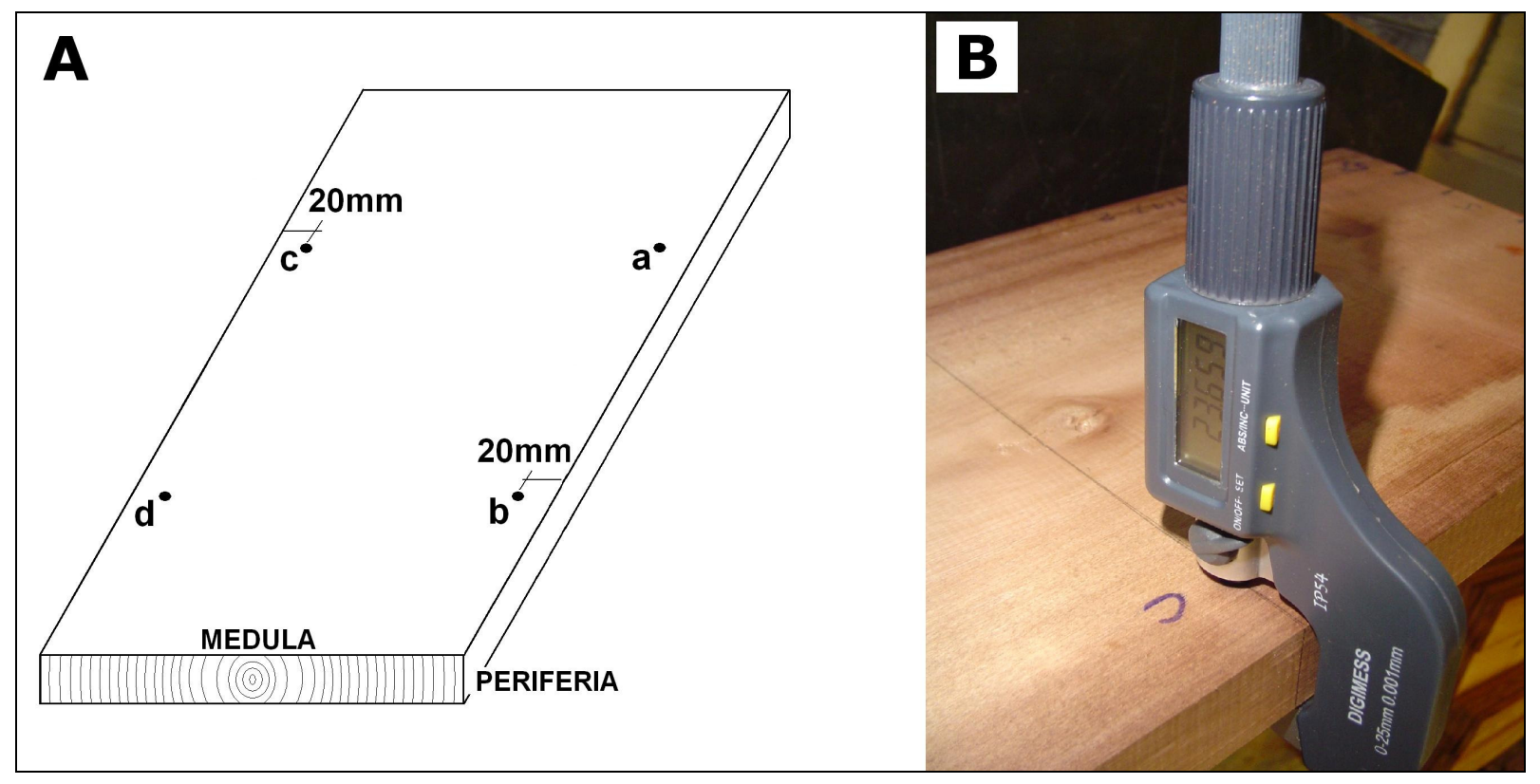

Figura 1 - Esquema sobre a posição dos pontos de medição da espessura das tábuas (A) e tábua de $E$. grandis com demarcação dos pontos de medição da espessura (B).

\section{RESULTADOS E DISCUSSÃO}

Para cada uma das condições empregadas na termorretificação da madeira de Eucalyptus grandis determinou-se a influência dos tratamentos na variação da espessura das tábuas.

Salienta-se que todas as tábuas de Eucalyptus grandis utilizadas no experimento foram obtidas de pranchas centrais. Então, em cada tábua foram realizadas medições das espessuras nas regiões próximas à periferia do fuste. 
A normalidade dos dados, da variação da espessura das tábuas após a termorretificação, recomendou a adoção de métodos de análise paramétrica (ANOVA) para análise de variância e a aplicação do teste de Tukey para comparação múltipla.

Assim, verifica-se, pela Tabela 2, que houve uma variação significativa ao nível de 5\% de significância na diferença da espessura com a diminuição das condições de termorretificação. Nos tratamentos com maiores condições de tempo e de temperatura houve um aumento médio da espessura das tábuas entre $0,698 \mathrm{~mm}$ e $0,874 \mathrm{~mm}$.

Tabela 2 - Efeito da condição da termorretificação na variação da espessura das tábuas de Eucalyptus

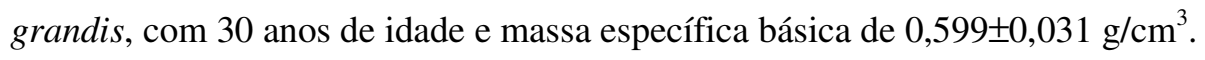

\begin{tabular}{ccccc}
\hline \multicolumn{2}{c}{ Termorretificação } & \multicolumn{2}{c}{ Variação da espessura da tábua } \\
\hline Temperatura $\left({ }^{\mathbf{0}} \mathbf{C}\right)$ & Tempo $(\mathbf{h})$ & $\mathbf{N}$ & $\Delta \mathbf{e}$ Média $(\mathbf{m m})$ & Tukey \\
\hline $\mathbf{2 0 0}$ & $\mathbf{4 , 0}$ & 10 & 0,698 & $\mathrm{a}$ \\
\hline $\mathbf{2 0 0}$ & $\mathbf{3 , 0}$ & 16 & 0,874 & $\mathrm{a}$ \\
\hline $\mathbf{1 8 0}$ & $\mathbf{2 , 5}$ & 14 & $-0,177$ & $\mathrm{~b}$ \\
\hline
\end{tabular}

Sendo: N - número de repetições; $\Delta$ e média - diferença média da espessura das tábuas; letras diferentes - indicam diferença significativa ao nível de 5\% de significância pelo teste de Tukey.

Era esperado, com base na literatura, uma redução mais pronunciada da espessura da tábua com o aumento do tempo e da temperatura do tratamento, pois a diminuição das dimensões está associada com a perda de massa e Alén et al. (2002), Juodeikiené e Minelga (2003), Hakkou et al. (2005a), Hakkou et al. (2005b), Metsä-Kortelainen et al. (2005), Unsal e Ayrilmis (2005) e Sundqvist et al. (2006) salientaram que as perdas de massa são mais pronunciadas com o aumento das condições do tratamento térmico.

Contudo, o aumento da espessura das tábuas com o aumento do tempo e da temperatura de termorretificação da madeira pode ser explicado pela presença de rachaduras internas no material termorretificado com temperatura de $200^{\circ} \mathrm{C}$, durante 3 a 4 horas. Verifica-se, que o aumento em espessura $(0,698$ $\mathrm{mm}$ ) nas tábuas termorretificadas à $200^{\circ} \mathrm{C}$, durante 4 horas, apresentou-se ligeiramente menor do que nas tábuas termorretificadas na mesma temperatura por 3 horas $(0,874 \mathrm{~mm})$. Uma das prováveis explicações para esse comportamento é que a madeira termorretificada por uma hora mais, apresentou uma maior degradação térmica o que, consequentemente, minimizou o aumento em espessura das tábuas mas, não impediu a expansão das rachaduras no interior das peças de madeira. 
A realização de cortes longitudinais ao longo dos pontos de medição de espessura das tábuas mostra que todo o lote do material termorretificado a $200^{\circ} \mathrm{C}$, durante 3 a 4 horas, apresentou rachaduras internas, e consequentemente, aumento nas suas espessuras (Figura 2).

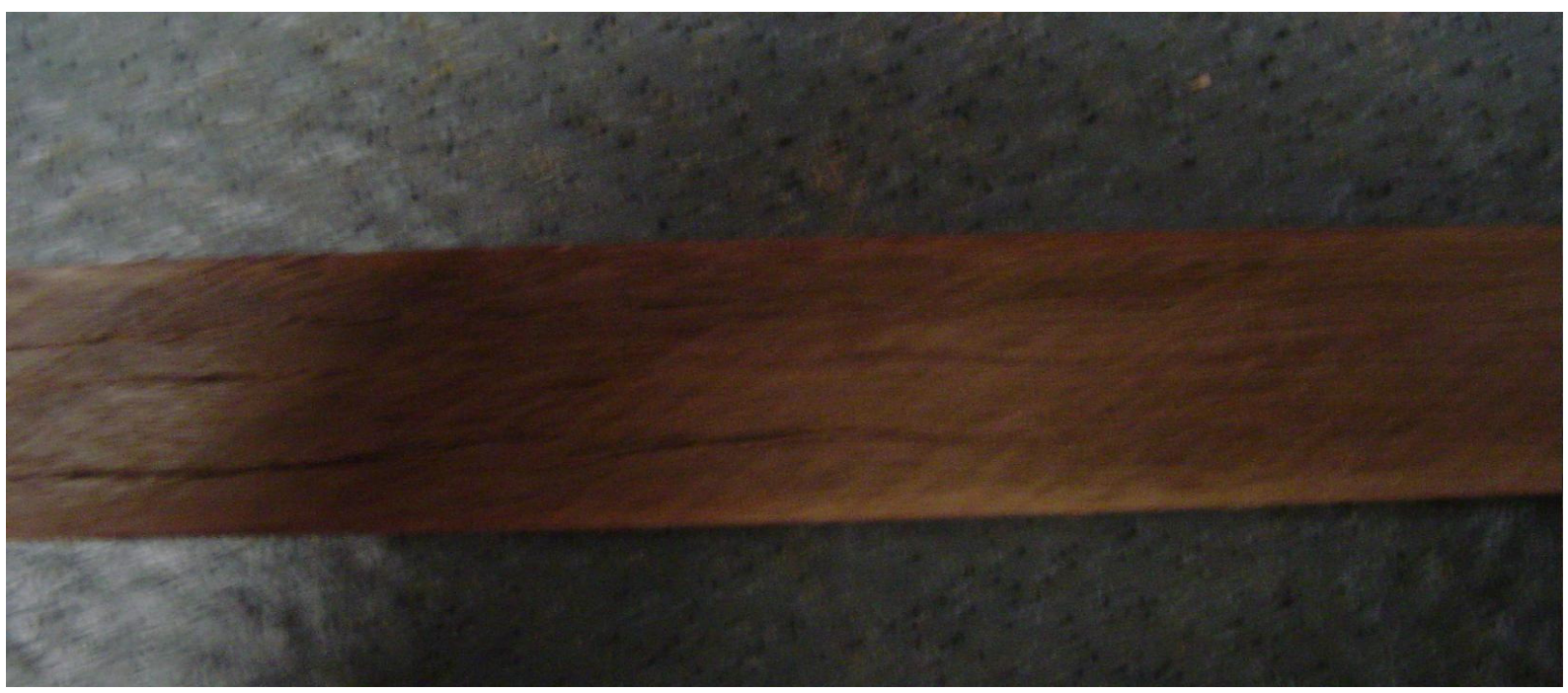

Figura 2 - Rachaduras internas ao longo de uma tábua de Eucalyptus grandis termorretificada com temperatura de $200^{\circ} \mathrm{C}$, durante 3 horas.

Somente a madeira de Eucalyptus grandis, com 30 anos e massa específica básica de $0,600 \mathrm{~g} / \mathrm{cm}^{3}$, quando termorretificada com temperatura de $180^{\circ} \mathrm{C}$ e durante um período de 2,5 horas é que apresentou uma redução na espessura média das suas peças em torno de $0,177 \mathrm{~mm}$. Após a realização de cortes ao longo dessas peças ficou constatado a ausência de rachaduras internas visíveis. Resultado semelhante foi apresentado por Rousset et al. (2004) que na termorretificação da madeira de Populus robusta à $200^{\circ} \mathrm{C}$, durante 1 hora, não houve alterações na permeabilidade da madeira e na morfologia dos poros e não ocorreram rachaduras internas.

\section{CONCLUSÕES}

Sobre o efeito do tempo e da temperatura de termorretificação sobre a espessura das tábuas de Eucalyptus grandis, concluiu-se que:

a) o material termorretificado com $200^{\circ} \mathrm{C}$ durante 3,0 a 4,0 horas apresentou aumento da espessura entre 0,698 e $0,874 \mathrm{~mm}$ devido ao surgimento de rachaduras internas;

b) o material termorretificado com $180^{\circ} \mathrm{C}$ durante 2,5 horas apresentou diminuição de $0,177 \mathrm{~mm}$ na sua espessura e não ocorreu presença de rachaduras internas. 


\section{REFERÊNCIAS}

ALÉN, R.; KOTILAINEN, R.; ZAMAN, A. Thermochemical behavior of Norway spruce (Picea abies) at 180-225 ${ }^{\circ}$. Wood Science and Technology, Berlin, v. 36, n. 2, p. 163-171, 2002.

BEKHTA, P.; NIEMZ, P. Effect of high temperature on the change in color, dimensional stability and mechanical properties of spruce wood. Holzforschung, Berlin, v. 57, p. 539-546, 2003.

BRITO, J.O.; GARCIA, J.N.; BORTOLETTO JUNIOR, G. Densidade básica e retratibilidade da madeira de Eucalyptus grandis submetida a diferentes temperaturas de termorretificação. Cerne, Lavras, v. 12, n. 2, p. 182-188, 2006.

HAKKOU, M.; PÉTRISSANS, M.; EL BAKALI, I.; GÉRARDIN, P.; ZOULALIAN, A. Wettability changes and mass loss during heat treatment of wood. Holzforschung, Berlin, v. 59, p. 35$37,2005 b$.

HAKKOU, M.; PÉTRISSANS, M.; ZOULALIAN, A.; GÉRARDIN, P. Investigations of wood wettability changes during heat treatment on the basis of chemical analysis. Polymer Degradation and Stability, Amsterdam, v. 89, n. 1, p. 1-5, 2005a.

JUODEIKIENĖ, I.; MINELGA, D. The influence of heating on wood hygroscopicity and dimensional stability. Materials Science (Medziagotyra), Kaunas, v. 9, n. 2, p. 209-212, 2003.

METSÄ-KORTELAINEN, S.; ANITIKAINEN, T.; VIITANIEMI, P. The water absorption of sapwood and heartwood of Scots pines and Norway spruce heat-treated at $170^{\circ} \mathrm{C}, 190^{\circ} \mathrm{C}, 210^{\circ} \mathrm{C}$ and $230^{\circ} \mathrm{C}$. Holz als Roh- und Werkstoff, Berlin, v. 64, n. 3, p. 192-197, 2005.

POPPER, R.; NIEMZ, P.; EBERLE, G. Untersuchungen zum Sorptions- und Quellungsverhalten von thermisch behandeltem Holz. Holz als Roh- und Werkstoff, Berlin, v. 63, n. 2, p. 135-148, 2005. 
RAPP, A. O. Review on heat treatments of wood. In: SPECIAL SEMINAR ENVIRONMENTAL OPTIMISATION OF WOOD PROTECTION, 1, 2001. Antibes, FRA. Proceedings... Antibes, FRA: COST ACTION E 22, 2001. 68p.

ROUSSET, P.; PERRÉ, P.; GIRARD, P. Modification of mass transfer properties in poplar wood (P. robusta) by thermal treatment at high temperature. Holz als Roh- und Werkstoff, Berlin, v. 62, n. 2, p. 113-119, 2004.

SUNDQVIST, B.; KARLSSON, O.; WESTERMARK, U. Determination of formic-acid and acetic acid concentrations formed during hydrothermal treatment of birch wood and its relation to colour, strength and hardness. Wood Science and Technology, Berlin, v. 40, n. 7, p. 549-561, 2006.

UNSAL, O; AYRILMIS, N. Variations in compression strength and surface roughness of heattreated Turkish river red gum (Eucalyptus camaldulensis) wood. Journal Wood Science, Tóquio, v. 51, n. 4, p. 405-409, 2005.

WASKETT, P.; SELMES, R. E. Opportunities for UK grown timber: wood modification state of the art review. Garston/Watford. Building Research Establishment, 2001. 83 p. Projeto n. 203343. 